\title{
The involvement of illusory contours in stroboscopic motion
}

\author{
MICHAEL W. von GRÜNAU \\ Max-Planck-Institut für Psychiatrie, Kraepelinstrasse 2, 8000 München 40, West Germany
}

\begin{abstract}
It was tested whether illusory contours, as they appear in the Ehrenstein figure, are able to contribute to stroboscopic motion in addition to the contribution of their inducing (real) contours. It was found that form information associated with illusory contours can be used by the stroboscopic motion mechanism. This is evidence that illusory contours can have functional effects that resemble those of real contours.
\end{abstract}

Stroboscopic motion experiences occur when two spatially separated stimuli are flashed briefly in close succession. One usually observes a single object moving from the position of the first stimulus to that of the second. The involvement of contour information in the generation of these experiences of movement is not clear. It has been suggested that the important point is the difference in location of the two inducing stimuli and that motion is perceived in order to bridge this disparity (e.g., Wertheimer, 1912). In this model, contour or form information plays only a subordinate role. On the other hand, perception of figures at disparate locations was regarded as a necessary prerequisite to the perception of motion (e.g., van der Waals \& Roelofs, 1930). Other theoretical positions lie in between these extremes, stating that descriptions based solely on figure perception or solely on motion perception cannot account for all the facts. Thus, Kolers (1972) suggests the operation of two analytically separable systems with different thresholds, one for motion, the other for figure. More recently, a similar idea was expressed by Pantle and Picciano (1976), who proposed two separate motion systems, one being independent of form and operating at short intervals between the inducing flashes, the other being dependent on form and operating at longer intervals. While the prior conscious perception of the form of the inducing stimuli cannot be a necessary condition for the occurrence of motion, stroboscopic motion experiences seem to be tied closely to the processing of contour information provided by the inducing stimuli. On the other hand, motion does still occur quite readily, even if one of the inducing stimuli is completely masked (Kolers, 1963; von Grünau, 1978). On the other hand, when contour information is available from both stimuli, both

This research was partially supported by funds from the Deutsche Forschungsgemeinschaft, SFB 50, Kybernetik. I wish to thank Dr. W. Singer for his most generous support and valuable advice, and L.W. for being a very patient observer. Reprints may be obtained from the author at Department of Psychology, Queen's University, Kingston, Ontario K7L 3N6, Canada. seem to contribute to the form of the apparently moving object in the space between the two stimulus flashes. Thus, when the two stimuli have different shapes, the shape of the apparently moving object changes smoothly from the shape of the first stimulus to that of the second as it moves (Kolers \& von Grünau, 1976).

Usually, contours are defined by a border between two areas of different luminances. There also exist, however, illusory contours which appear within areas with a homogeneous luminance distribution, induced by certain arrangements of real contours (Kanizsa, 1955; Schumann, 1904). Various underlying mechanisms have been suggested, such as simultaneous brightness contrast (Frisby \& Clatworthy, 1975; Jung \& Spillman, 1970), the processing of depth (Coren, 1972), and perceptual hypotheses (Gregory, 1972). The place of processing within the visual system, therefore, has been localized as peripherally as the retina and as centrally as higher cognitive centers. In order to get a better understanding of the nature of these illusory contours, several investigators have tested whether illusory contours produce geometrical illusions. It was demonstrated, for example, by Gregory (1972) that the Poggendorff illusion seems to be caused by illusory contours in a way similar to that caused by real contours.

Following this line of inquiry, it was the purpose of the present study to find out whether illusory contours could contribute to stroboscopic motion in a way that was different from or in addition to the contribution of their inducing contours. In other words, can illusory contours provide form information that the stroboscopic motion mechanism can use to generate a moving object? The results show that form information associated with illusory contours can be used by the stroboscopic motion mechanism in a way similar to form information associated with real contours.

\section{METHOD}

Illusory contours exist only in connection with their inducing (real) contours. A basic difficulty in assessing stroboscopic motion 


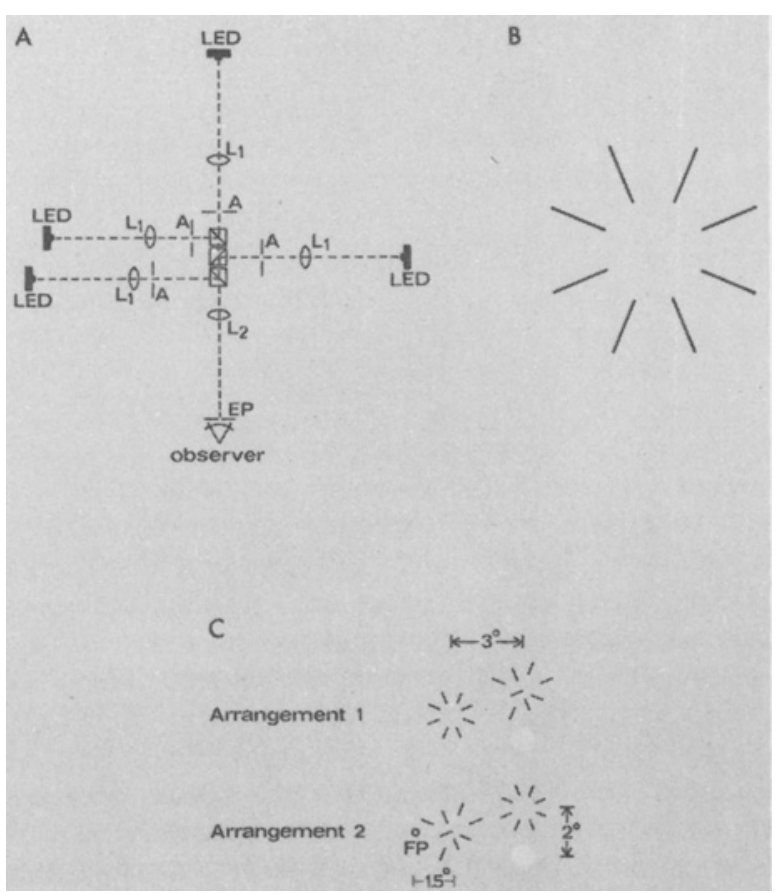

Figure 1. (A) Schematic representation of the four-channel Maxwellian view system. Light from the LEDs is collimated by lenses $L_{1}$. Images of apertures $A$ are formed in the plane of the exit pupil (EP) by lens $L_{2}$. (B) Ehrenstein figure. An apparent light disk is seen in the center. (C) Stimulus arrangements. The left figure and the two figures at the right were briefly flashed in alternation to produce stroboscopic motion.

of illusory contours, therefore, is to separate motion of the inducing contours from motion of the illusory contours. The basic figure used here was the Ehrenstein figure (Ehrenstein, 1941; Spillmann, 1977). When it is drawn with black lines on a light background, illusory contours appear in such a way that an apparent disk is seen in the center, and this disk seems brighter than the background (Figure 1B). The real-disk figure had a diameter of $1^{\circ}$ and appeared with an illumination of 1.8 lux on a background of 1.5 lux. The Ehrenstein figure consisted of $.5^{\circ}$-long black lines arranged to form an apparent disk with a diameter of $1^{\circ}$. The real and apparent disks appeared to have approximately equal brightness.

The rationale of the experiment was to compare the probability of seeing motion of a disk for two conditions: first, when an apparent disk (Ehrenstein figure) is alternated with a real disk, and second, when an "irregular star" figure is alternated with a real disk. The "irregular star" consisted of the same inducing lines as the Ehrenstein figure, but these were arranged haphazardly and thus did not produce apparent contours forming an apparent disk. A greater probability in the first case would indicate an involvement of the illusory contours above that of the inducing lines.

\section{Apparatus}

A four-channel Maxwellian view system with red LEDs as light sources was used to present the stimulus displays. The observer watched the displays with his right eye through a 2-mm-diam exit pupil, his head position being stabilized by individually fitted bite bars. The timing and intensity of the LEDs were controlled by electronic circuitry. Square-wave flashes of light with steady-state illumination of 1.5 lux illuminated photo- graphic apertures which determined the spatial properties of the stimuli. The observer fixated a small fixation point (FP). A schematic representation of the apparatus is given in Figure 1A.

\section{Stimuli}

To achieve the perception of stroboscopic motion, two briefly flashed stimuli (100 or $200 \mathrm{msec}$ each) were alternated with a variable interstimulus interval (ISI) between the end of one and the beginning of the other stimulus (ranging from 0 to $300 \mathrm{msec}$ ). Apparent disk, irregular star, and real disk were alternated in various combinations. Subjective reports and quantitative pilot studies showed that between the apparent disk and the irregular star, movement of black lines only was observed. Motion of a disk was never reported. Between the apparent disk and the real disk, on the other hand, motion was always of a disk and never of black lines. These seemed to flash on and off only. Between the irregular star and the real disk, the clearly dominating percept was motion of a disk. Only about $13 \%$ of the observed motion was motion of black lines.

It was found that the same kinds of motion experiences occurred when the figures were arranged as indicated in Figure 1C. The left stimulus consisted of one figure, either the apparent disk (Arrangement 1) or the irregular star (Arrangement 2). The right stimulus, separated from the left stimulus by $3^{\circ}$, consisted of two figures, either the irregular star and the real disk (Arrangement 1) or the apparent disk and the real disk (Arrangement 2). In each case, the two figures were separated by $2^{\circ}$ and arranged vertically. A fixation point was provided $1.5^{\circ}$ to the left of the left stimulus.

With appropriate timing, arrangements of this sort have been shown to result in the perception of apparent motion between the left stimulus figure and either one or both of the two figures of the right stimulus (Kolers, 1972; Wertheimer, 1912). The latter condition, in which motion occurs along two paths simultaneously, is sometimes referred to as "split motion". In Arrangement 1, motion could thus be seen between the apparent disk and either the irregular star (motion of black lines) or the real disk (motion of a disk), or both simultaneously. In Arrangement 2 , motion occurred similarly between the irregular star and either one or both of the two figures of the right stimulus: the apparent disk (motion of black lines) and the real disk (motion of a disk). These particular arrangements allowed the assessment of motion of a disk when illusory contours were or were not present, in relation to the same comparison motion. This motion of black lines was sufficiently different to permit clear differentiation of the two percepts.

\section{Procedure}

The four conditions, defined by the combinations of the two arrangements with the two exposure conditions, were presented in randomized order three times each within each of two sessions. Within each condition, one randomized set of six ISIs was presented, and the stimuli in any trial were recycled at a fixed ISI for $30 \mathrm{sec}$, so as to produce back-and-forth stroboscopic motion. In Session 1, the figures of the right stimulus flash were arranged as shown in Figure 1C, and in Session 2, top and bottom figures were exchanged.

Before each session, the observer was dark-adapted for 20 min and then briefly light-adapted to the background level of illumination. He was to respond only when he was looking at the fixation point. The observer's task was to indicate, by pressing the appropriate key, whenever he perceived motion between the left stimulus and the upper and/or the lower figure of the right stimulus. His responses were recorded on a two-channel polygraph recorder.

In each arrangement, duration of the motion of a disk was compared to duration of the motion of black lines. Furthermore, the latency with which motion of a disk or motion of black lines first occurred within a presentation period was measured.

The two observers had normal color and spatial vision, as 
assessed with H-R-R Pseudoisochromatic Plates and the Bausch \& Lomb Orthorater, and were well acquainted with psychophysical experiments. Both were well practiced in the task of this experiment, and L.W. was completely unaware of the purpose of the present study. The results were analyzed using analysisof-variance procedures.

\section{RESULTS}

Both observers reported consistently that motion of a disk was seen between, for example, the apparent disk and the real disk, while motion of black lines was seen between, for example, the irregular star and the apparent disk. Both types of motion were also seen simultaneously (split motion). In Arrangement 1, for instance, black lines moved between the apparent disk and the irregular star, while a disk moved between the apparent disk and the real disk. In Figure 2, average duration of observed motion during a presentation period for the four possible combinations of figures is plotted as a function of ISI for a stimulus duration of $100 \mathrm{msec}$ in A and B, and a duration of $200 \mathrm{msec}$ in C and D. Overall, motion occurs for somewhat longer periods when the exposure duration is longer $(\mathrm{p}<.01)$, and motion of black lines is more likely to occur than motion of a disk $(\mathrm{p}<.01)$.

The main purpose of this experiment, however, was to compare motion of a disk when illusory contours were present and when they were not present in one of the inducing stimuli. When the stimulus duration was $100 \mathrm{msec}$, observer M.G. showed significantly more $(p<.01)$ motion for the case when illusory contours were present (apparent disk-real disk). Observer L.W., however, showed no significant difference $(p>.05)$ between the two cases. When exposure duration was increased to $200 \mathrm{msec}$, both observers reported significantly more motion in the case where illusory contours forming an apparent disk were present ( $p<.05$ for L.W.; $p<.01$ for M.G.) than where they were not. This result is summarized in Figure 2, E and F. The duration of abserved motion, averaged over ISI is plotted for motion of a disk when an irregular star is alternated with a real disk and when an apparent disk is alternated with a real disk. The presence of illusory contours significantly increases the amount of observed motion.

The results from the latency measurements (see Table 1) are in line with the above results, if it is assumed that a short latency indicates that the corresponding motion occurs more readily than motion which has a long latency. That is, latency of the first occurrence of motion is taken as an indicator of the ease with which the stroboscopic motion mechanism can construct motion. According to this, motion of a disk in the present situation is a more complicated task than motion of the black lines. Since latency for motion of a disk is shorter when illusory contours are present than when they are not, the presence of illusory contours in this case seems to enhance the ability of the stroboscopic motion mechanism to construct motion of a disk. Again illusory contours are found to contribute to stroboscopic motion.

\section{DISCUSSION}

In stroboscopic motion, contours are flashed briefly at two disparate locations. Information about these contours is used by the stroboscopic motion

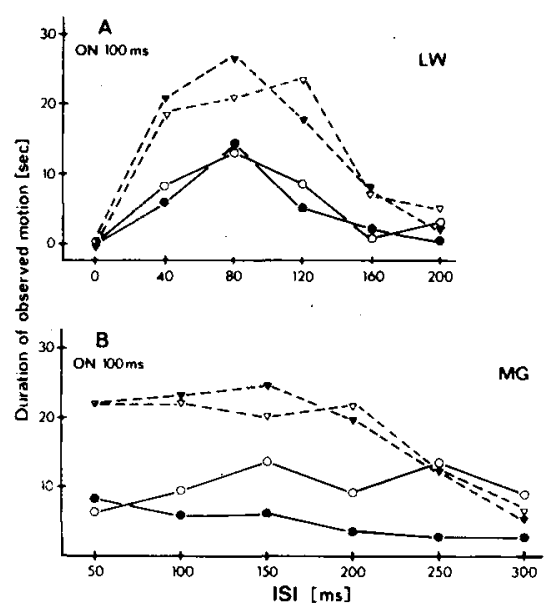

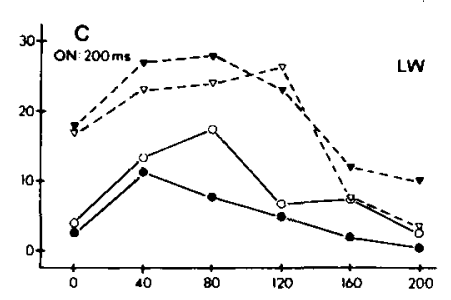

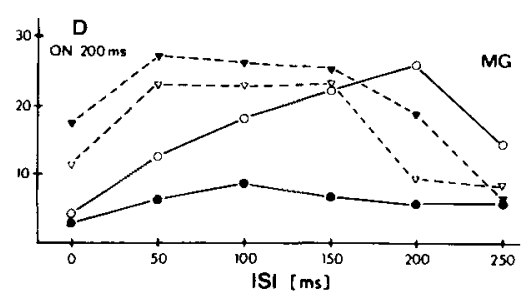

$\left.\begin{array}{l}\text { apparent disk } \ldots \text { irregular star } \\ \text { irregular star apparent disk }\end{array}\right\}$ motion of black lines
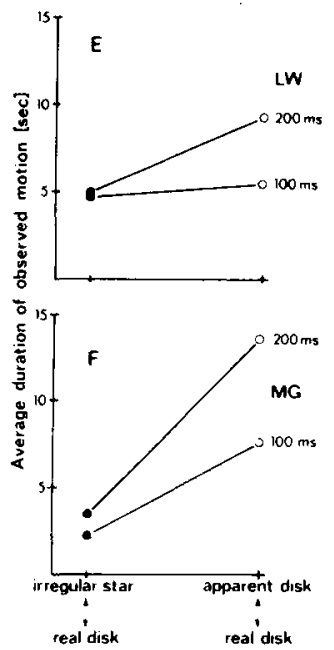

Figure 2. (A and B) Duration of observed motion as a function of interstimulus interval for observers L.W. and M.G. at an expo: sure duration of 100 msec. Open symbols, Arrangement 1; filled symbols, Arrangement 2. (C and D) Same as A and B, but at an exposure duration of $200 \mathrm{msec}$. (E and F) Average duration of observed motion for two combinations of inducing stimuli with exposure duration as parameter. 
Table 1

Latencies (in Milliseconds) for the First Occurrence of Motion with \pm 1 Standard Deviation

\begin{tabular}{lrrrrr}
\hline & \multicolumn{4}{c}{ Latency } \\
\cline { 2 - 4 } & \multicolumn{2}{c}{100} & & \multicolumn{2}{c}{200} \\
\cline { 2 - 3 } \cline { 5 - 6 } & Mean & SD & & Mean & SD \\
\hline Apparent Disk $\leftrightarrow$ Irregular Star & 5.3 & 3.4 & & 4.2 & 3.0 \\
L.W. Irregular Star $\leftrightarrow$ Apparent Disk & 5.6 & 6.0 & 3.8 & 2.9 \\
Apparent Disk $\leftrightarrow$ Real Disk & 10.8 & 5.4 & 11.6 & 4.3 \\
Irregular Star $\leftrightarrow$ Real Disk & 14.0 & 7.6 & 13.9 & 6.2 \\
Apparent Disk $\leftrightarrow$ Irregular Star & 2.7 & 2.8 & & 3.7 & 4.3 \\
M.G. Irregular Star $\leftrightarrow$ Apparent Disk & 3.5 & 6.4 & & 3.0 & 3.3 \\
Apparent Disk $\leftrightarrow$ Real Disk & 10.6 & 4.8 & 6.9 & 2.9 \\
Irregular Star $\leftrightarrow$ Real Disk & 15.9 & 6.6 & 12.6 & 1.9 \\
\hline
\end{tabular}

mechanism to produce an apparent object which moves between two stimulus locations. In the present experiment, it was tested whether illusory contours as part of one of the stimulus flashes can provide contour information which can contribute to stroboscopic motion. It was shown that a stimulus containing illusory contours can lead to more stroboscopic motion than a similar stimulus which does not contain illusory contours. It is therefore concluded that illusory contours are able to supply contour information to the stroboscopic motion mechanism for the construction of the moving object's form.

This ability, however, depends on the presentation time of the inducing stimulus flashes. It was shown here that a longer duration of the inducing stimuli increases the effectiveness of illusory contours. In this respect, it is already known that the occurrence of illusory contours depends on the duration of the inducing stimuli. Spillmann, Fuld, and Gerrits (1976) report that some brightness enhancement, and the corresponding illusory contours, were present in the Ehrenstein figure with exposure durations of only $30 \mathrm{msec}$. This value shows individual differences and also depends on the particular arrangement, its contrast, size, etc. It is, therefore, reasonable to assume that illusory contours were weak or not consistently present for observer L.W. when the duration was only $100 \mathrm{msec}$. An effect of the illusory contours therefore did not show up. When the duration was increased to $200 \mathrm{msec}$, this observer, too, showed a sizable effect.

The fact that relatively long exposure times were necessary before illusory contours could contribute to stroboscopic motion might indicate that a process analogous to the figure formation of real contours (Kolers, 1964) is operating with illusory contours. However, the time constants involved seem to be longer than those for real contours.

The study of other functional effects of illusory contours, such as their involvement in geometrical illusions, has not been able to clarify the extent to which illusory contours can actually influence real contours. Though strong illusions are usually found (Gregory, 1972, 1975; Pastore, 1971), these may be caused by factors unrelated to contours (Day, Dickinson, \& Jory, 1977; Kanizsa, 1974). The present results, that illusory contours can interact with real contours in a stroboscopic motion situation, provide some evidence that illusory contours can have functional effects that resemble those of real contours. It remains to be shown, however, that stroboscopic motion can also occur between two sets of illusory contours. Only this could establish their functional equivalence to real contours.

\section{REFERENCES}

Coren, S. Subjective contours and apparent depth. Psychological Review, 1972, 79, 359-367.

DAY, R. H., Dickinson, R. G., \& JoRY, M. K. The Poggendorff illusion with subjective contours. Quarterly Journal of Experimental Psychology, 1977, 29, 219-226.

ERRENSTEIN, W. Über Abwandlungen der L. Hermannschen Helligkeitserscheinung. Zeitschrift für Psychologie, 1941, 150, 83-91.

FrISBy, J. P., \& Clatworthy, J. L. Illusory contours: Curious cases of simultaneous brightness contrast? Perception, 1975, 4, 349-357.

GREGORY, R. L. Cognitive contours. Nature, 1972, 238, 51-52.

GREGoRY, R. L. Illusion-destruction by appropriate scaling. Perception, 1975, 4, 203-220.

Jung, R., \& Spillmann, L. Receptive field estimation and perceptual integration in human vision. In F. A. Young \& D. B. Lindsley (Eds.), Early experience and visual information in perceptual and reading disorders. Washington, D. C: National Academy of Sciences, 1970. Pp. 181-197.

Kanzsa, G. Margini quasi-percettivi in campi con stimulazione omogenea. Rivista di Psicologia, 1955, 49, 7-30.

KanIzSA, G. Contours without gradients or cognitive contours? Giornale Italiano di Psicologia, 1974, 1, 93-112.

Kolers, P. A. Some differences between real and apparent visual movement. Vision Research, 1963, 3, 191-206.

Kolers, P. A. The illusion of movement. Scientific American, 1964, 211, 98-106.

Kolers, P. A. Aspects of motion perception. New York: Pergamon Press, 1972.

Kolers, P. A., \& von Grünau, M. W. Shape and color in apparent motion. Vision Research, 1976, 16, 329-335.

Pantle, A., \& Picciano, L. A multistable movement display: Evidence for two separate motion systems in human vision. Science, 1976, 193, 500-502.

PAstore, N. Selective history of theories of visual perception 1650-1950. New York: Oxford University Press, 1971.

Schumann, M. Einige Beobachtungen über die Zusammenfassung von Gesichtseindrücken zu Einheiten. Psychologische Studien, $1904,1,1-32$.

SPILlmaN, L. Kontrastsehen in Helligkeitstäuschungen. Habilitationsschrift, University of Freiburg, W. Germany, 1977.

Spillmann, L., Fuld, K., \& GerRits, H. J. M. Brightness contrast in the Ehrenstein illusion. Vision Research, 1976, 16, 713-719.

Van DeR WaAs, H. G., \& Roelofs, C. O. Optische Scheinbewegung. Zeitschrift für Psychologie und Physiologie der Sinnesorgane, 1930, 114, 241-288.

von GrïnaU, M. W. Dissociation and interaction of form and motion information in the human visual system. Vision Research, 1978, 18, 1485-1489.

Wertheimer, M. Experimentelle Studien über das Sehen von Bewegung. Zeitschrift für Psychologie, 1912, 61, 161-278.

(Received for publication June 1, 1978;

revision accepted December 7,1978 .) 